\title{
Commentary
}

\section{Novel coronavirus and hospital infection prevention: Preparing for the impromptu speech}

\author{
Gonzalo Bearman MD, MPH ${ }^{1}$, Rachel Pryor RN, MPH ${ }^{1}$ (1), Heather Albert RN, MSN ${ }^{1}$, Lisa Brath MD, FCCP², \\ Amy Britton RN, MSN ${ }^{1}$, Kaila Cooper RN, MSN ${ }^{1}$, Michelle Doll MD, $\mathrm{MPH}^{1}$, Emily J Godbout DO, MPH ${ }^{3}$, \\ Robin Hemphill MD, MPH ${ }^{1}$ and Michael P Stevens MD, MPH ${ }^{1}$ \\ ${ }^{1}$ Healthcare Infection Prevention Department, Virginia Commonwealth University Health System, Richmond, Virginia, ${ }^{2}$ Department of Medicine, Virginia \\ Commonwealth University Health System, Richmond, Virginia and ${ }^{3}$ Division of Pediatric Infectious Diseases, Department of Pediatrics, Children's Hospital of \\ Richmond at Virginia Commonwealth University, Richmond, Virginia
}

"It usually takes me two or three days to prepare an impromptu speech." - Mark Twain

Once again we are put to the test. With the emergence of the 2019 novel human coronavirus (2019-nCoV), healthcare systems worldwide are scrambling to meet the demands of a growing outbreak. Much akin to the appearance of severe acute respiratory syndrome coronavirus (SARS CoV) and Middle East respiratory syndrome coronavirus (MERS CoV), the ultimate scope and reach of the outbreak is fluid and presently unknown. Unlike Ebola virus disease, with its high infectivity and high mortality, no uniform demand exists for assessment and treatment of 2019-nCoV infected patients in designated unique pathogen unit (UPU) settings. Regardless, the evolving understanding of transmission dynamics and the increasing international case load, coupled with growing fear and misinformation, results in formidable pressure on healthcare systems to maximize patient triage, staffing, confirmatory testing, communication, use of personal protective equipment (PPE), and patient placement. In the face of immense challenges, maximum and relentless standardization of base operational processes serves as the bedrock for nuanced, nimble responsiveness to real-time threats.

\section{Identifying the key stakeholders and standardizing communications}

Healthcare infection prevention programs (HIPPs) should identify stakeholders from the local health department, emergency department, microbiology, hospital leadership, emergency management, UPU team (if applicable), and both nursing and physician leaders a priori with clear expectations for whom to contact, when, and via what mechanisms. Collectively, these stakeholders must establish regular communication plans. The use of existing communication infrastructure within the healthcare system along with preproduced resources (eg, fact sheets and key messages) that can be tailored to the $2019-\mathrm{nCoV}$ will expedite information sharing. ${ }^{1}$

\footnotetext{
Author for correspondence: Rachel Pryor, E-mail: Rachel.pryor@vcuhealth.org

Cite this article: Bearman G, et al. (2020). Novel coronavirus and hospital infection prevention: Preparing for the impromptu speech. Infection Control \& Hospital Epidemiology, 41: 592-593, https://doi.org/10.1017/ice.2020.55

Similar to communications between health department and hospital leaders, communication with healthcare workers (HCW) should be explicit and the information provided should be frequent, focused, and current. Frequent communications ensure that HCWs feel they have the correct information, which can limit the spread of misinformation. The healthcare system's default posture should include the assumption that a novel pathogen is transmissible and poses a threat. Healthcare team members must feel that the HIPP and leadership are responding with both urgency and concern.

Efforts to quickly detect, respond to, and manage an outbreak depend largely on the availability of reliable communication infrastructure that can support the optimization of decision making for stakeholders. The establishment of preapproved, systematic protocols to guide decision making is critical. Teams that initiate communications must have the appropriate protocols, procedures, contact lists, and paging numbers to initiate coordination of communication that include up-to-date, off-hour contact details in the communications plan and set the expectation for frequency of follow-up during the initial communications.

Options to coordinate with key stakeholders range from e-mail communications and exchange of informational materials to joint decision-making meetings. These meetings need to be both reliable and flexible, allowing stakeholders to participate according to the specific nature of the situation. Teleconference or face-to-face meetings may be appropriate based on the specific challenge and the groups involved. Web-based, secure communication systems may also be helpful as a means of providing input and exchanging materials and information.

\section{To minimize chaos, make the management structure explicit}

A comprehensive all-hazards approach and a well-developed emergency preparedness plan are crucial to providing streamlined and consistent messaging. Equally essential is a predetermined, hierarchical chain of command that incorporates the local organizational structure to ensure a maximally organized response. Standardization and establishment of clear roles, expectations, and accountability for the leadership team members empowers each team member to participate in a fully organized and efficient 
response. A hierarchical management structure is also crucial to providing streamlined and consistent messaging. All of these mechanisms should be standardized and ready.

\section{Standardize patient triage, placement, and staffing models to the fullest extent possible}

Stakeholders must create a standardized process entailing rapid triage, isolation, and placement of patients with suspected 2019$\mathrm{nCoV}$ to minimize the risk of transmission and exposure of HCWs and other patients. Patient placement decisions should incorporate known characteristics of the organism as well as available resources. At a minimum, patient placement needs must incorporate specific guidelines regarding isolation and air handling.

For 2019-nCoV, current recommendations include a single occupancy, negative pressure room with at least 6 air changes per hour. ${ }^{2}$ Rooms designated as negative pressure rooms within an institution should be checked by a plant operations supervisor to ensure that the room is functioning as expected prior to receiving a patient. Entry and exit to the room should be minimized. If possible, healthcare facilities should consider a dedicated HCW staffing team to minimize the risk of transmission and exposure to other HCWs and patients. ${ }^{2}$ If this is not feasible, only essential HCW should enter the room. Detailed planning of staffing models will help alleviate potential staff shortages in the event of multiple suspect or infected patients needing care.

\section{Personal protective equipment supply and just in time training}

Personal protective equipment (PPE) recommendations for HCWs caring for possible or confirmed 2019-nCoV include gloves, gowns, eye protection and a mask. ${ }^{1,2}$ The Centers for Disease Control and Prevention (CDC) recommends the mask be a National Institute for Occupational Safety and Health (NIOSH)-certified N95 respirator or a reusable powered airpurifying respirator (PAPR). ${ }^{2}$ Unit leadership, with support from the HIPP and supply management and logistics, must visually confirm the supply of appropriate PPE on site. Mechanisms to retrieve additional PPE must be clear for all staff members in the unit, ideally prior to the arrival of a potentially infected patient.

"Just in time" refresher training will assist HCWs in the proper donning and doffing of PPE. This training can take the form of visual flyers, posters, and/or videos. The presence of a HIPP or UPU staff member with expert knowledge in PPE is invaluable. Given the known risks of self-contamination, particularly when doffing of $\mathrm{PPE},{ }^{3}$ a monitor to enforce PPE recommendations and observe for potential breaches is a critical resource ensuring both HCW and other patients are protected.

\section{Preparing for the impromptu speech}

Emergency preparedness for novel pathogens is akin to diligently preparing for an impromptu speech. Much like a talented jazz musician whose training in music theory and technique facilitates skillful improvisation, emergency response to novel infectious diseases begins with consistent and sustained process standardization via a comprehensive all-hazards approach. This allows for maximal facility in communication, triage, staffing, PPE use and patient placement. Practice standardization makes real-time, impromptu responsiveness and improvisation more nimble by minimizing the diversion of focus and energy on standard operational matters.

Emergency response standardization stems from prioritization. Healthcare infection prevention programs guide healthcare system responses to evolving infectious diseases threats. ${ }^{4}$ Only institutional leaders can provide the necessary resources and institutionalize emergency responses. Keeping leaders engaged is an ongoing challenge. ${ }^{4}$ The emergence of $2019-\mathrm{nCoV}$ is a potent reminder of the ongoing need for consistent funding, prioritization, and relentless simulation of emergency response mechanisms by healthcare systems, even in the face of competing interests. Our ethical mandate is for safe and reliable care, especially in the face of evolving threats; anything less undermines the public's trust.

\section{References}

1. Infection prevention and control during health care when novel coronavirus $(\mathrm{nCoV})$ infection is suspected, interim guidance. World Health Organization website. https://www.who.int/publications-detail/infection-prevention-andcontrol-during-health-care-when-novel-coronavirus-(ncov)-infection-issuspected-20200125. Updated January 25, 2020. Accessed February 5, 2020.

2. Interim infection prevention and control recommendations for patient with confirmed 2019 novel coronavirus $(2019-\mathrm{nCoV})$ or patients under investigation for 2019-nCoV in healthcare settings. Centers for Disease Control and Prevention website. https://www.cdc.gov/coronavirus/2019nCoV/hcp/infection-control.html. Updated February 3, 2020. Accessed February 5, 2020.

3. Osei-Bonsu K, Masroor N, Cooper K, et al. Alternative doffing strategies of personal protective equipment to prevent self-contamination in the health care setting. Am J Infect Control 2019;47:534-539.

4. Bearman GM, Vokes RA. Averting a betrayal of trust: system and individual accountability in healthcare infection prevention. Infect Control Hosp Epidemiol 2019;40:918-919. 